\title{
Towards in situ detection of PAH trace in sea-water using SERS-active sensors
}

\author{
Olivier Péron, Emmanuel Rinnert*, Michel Lehaitre, Florent Colas, Chantal Compère \\ IFREMER, Service Interfaces et Capteurs, BP 7029280 Plouzané, France
}

\begin{abstract}
This paper reports the development of a sensor based on surface-enhanced Raman scattering (SERS) for analyses in seawater. Polycyclic aromatic hydrocarbons (PAHs) are targeted by these sensors and their detection in situ summons up chemical synthesis and optical development. Firstly, a relevant synthesis of SERS active substrates based on gold nanostructures is presented. Different kinds of substrates have been synthesized under variable experimental conditions to modify some parameters such as i) gold shape, size and distribution and such as ii) chemical functionalization: (i) gold nanoparticles were prepared either by chemical reduction of $\mathrm{HAuCl}_{4}$ or by physical deposition. (ii) Substrates were functionalized by hydrophobic films to allow nonpolar molecules pre-concentration. Low concentration from ppb to ppm of PAHs were detected with a Raman microscope designed for lab experiments. Sensors exhibit strong enhancement of Raman scattering from molecules adsorbed on the films. Spectra were recorded for two PAHs (naphthalene and pyrene) in artificial sea-water with limits of detection of 10ppb for both with a short integration time (10s) and a low incident laser power $(\sim 0.1 \mathrm{~mW})$. Active substrate surface morphology was characterized with scanning electron microscopy (SEM) measurements. Secondly, an home-made in situ Raman spectrometer was developed and has been connected to a micro-fluidic system. This system was designed to host SERS-active sensors in order to ensure measurements with a flow cell. This original configuration of in situ Raman spectroscopy was then achieved. Such a device is now ready to use to confirm the PAH detection at ppb levels during the offshore experiments thanks to SERS sensors.
\end{abstract}

Keywords: functionalization, silanization, gold nanostructures, SERS, PAH detection, sea-water, sensor, in situ.

\section{INTRODUCTION}

Raman scattering is a spectroscopic technique which allows the identification of a wide variety of chemical and biological compounds through well defined vibrational informations. For the in situ sea-water analysis, the use of a such technique finds also its interest in the absence of the strong IR absorption band of the water. However, Raman scattering presents a very poor efficiency reducing the analyte detection at high concentration levels. In the investigation of chemical pollutions in sea-water, such as polycyclic aromatic hydrocarbon (PAH) trace concentration, sensitive sensors based on the surface-enhanced Raman scattering (SERS) effect can be used and be an alternative to the low cross-section of Raman scattering.

SERS is a very sensitive technique and considerable works have been performed with the aim to study a wide range of molecules. Different types of surfaces like colloidal metal nanoparticles, roughened metal foils, metal nanoparticle arrays obtained by electron beam lithography (EBL) were employed to play the role of the nanoantenna. The long-range electromagnetic model and the short-range chemical model [1-3] explain the Raman enhancement origins. The first one, which contributes it predominately, is related to the excitation of surface plasmons leading to an increase of the local electromagnetic field. The second one is linked to a temporary charge transfer between the adsorbate and the metal with a modification of the Raman polarizability tensor of the adsorbed molecule. The coupling of both mechanisms sparks off huge Raman enhancements with the SERS signals up to $10^{6}$ fold [4-6] the normal Raman intensity of the non-adsorbed molecules. More recently, enhancement factors of up to $10^{14}-10^{20}$ were obtained in single-molecule detection experiments [7-10]. Although silver metal presents a higher enhancement factor, 10-100 fold greater than gold metal, Au was chosen here on account of $\mathrm{AgCl}$ formation on silver surfaces in the marine environment [11]. 
In order to obtain a sensitive sensor to the hydrophobic molecules, such as PAHs, the strategy was to make substrate surfaces suitable for nonpolar molecule pre-concentration. Consequently PAHs, such as naphthalene and pyrene, are preferentially adsorbed on the sensor surface. The detection based on sol-gel process with Au or Ag nanoparticles embedded in a methyl/ethyl-tetraethoxysilane precursor has been done in previous works [12,13] through the EU funded MISPEC project. By this way, a limit of detection (LOD) of 430ppb naphthalene concentration was notified [13] and the LODs of $37.5 \mu \mathrm{M}$ and $0.1 \mu \mathrm{M}$, for naphthalene and pyrene respectively, in a sea-water solution of five PAHs was determined [14]. In this study, the bare substrate surfaces were functionalized with (3-mercaptopropyl)trimethoxysilane (MPMS) and (3-aminopropyl)trimethoxysilane (APTMS). The properties of functionalized substrate are modified. Indeed the nonpolar methylene groups of the MPMS or APTMS alkyl chains induce a hydrophobic behavior of the sensor surfaces. Moreover, molecular compounds with the $-\mathrm{SH}$ or $-\mathrm{NH}_{2}$ terminal groups have an affinity for the metal particles [15-17]. The MPMS and APTMS compounds allow both the hydrophobic pre-concentration of the nonpolar molecules and the covalent bonds with the gold nanoparticles.

\section{EXPERIMENTAL}

\subsection{Instrumentation}

SERS spectra of naphthalene and pyrene solutions were recorded at room temperature with a Raman spectrometer (Labram HR800-Jobin Yvon) using the 632.8nm excitation line of a He-Ne laser (power 0.1mW at the sample) and the 784.34nm excitation line of a laser diode (power $\sim 1 \mathrm{~mW}$ at the sample) in retrodiffusion geometry. The laser beam was focused through an Olympus water immersion lens $(x 100, N A=1)$ to a spot of around $1 \mu m^{2}$. Scattered radiation was collected at $180^{\circ}$ relative to the excitation beam and detected with an Andor CCD cooled by the Peltier effect. A $300 \mathrm{~g} / \mathrm{mm}$ grating coupled to a $800 \mathrm{~mm}$ spectrograph allows a spectral resolution of around $2 \mathrm{~cm}^{-1}$. Spectral calibration was performed on silicon samples (at $520 \mathrm{~cm}^{-1}$ ). Two stage edge filters allow Raman Stokes studies. The accumulation times were $5 \mathrm{~s}$ or $10 \mathrm{~s}$. The measurements were performed directly through a drop of the analyzed solution at the sensor surface.

Besides, a Raman spectrometer for in situ analysis was developed in collaboration with Jobin Yvon (EU funded Hermes project). The global set-up of the Raman system consists in a polyethylene frame on which both containers will be described later and the probe are settled (Figure 1A). This configuration is more convenient for shipboard interventions. Due to the flexibility of the optical fibre link, Raman probe can be settled in any orientation and it is fixed on the vertical position so that the flow through cell is more easily accessible without removing any part of the system. Thanks to this configuration, SERS substrates are easy to load (Figure 1B). The in situ Raman spectrometer It consists of three modules (Figure 2). The first one - the driving module - contains electronic boards and an industrial PC. It enables one to drive the spectrometer from the sea surface. The second module - the spectrometer module - holds an axial spectrograph coupled to a CCD camera (iDus, Andor) and a Laser diode of 785nm wavelength (BRM-785, B\&WTEK). Optical fibers link it to the third module - the SERS optode - where the SERS substrates can be placed. The spectrometer and the driving modules are made of anodized aluminium whereas the optode is of stainless steel. The whole instrument was qualified for use down to 5000m. Figure 3 shows the optical path within the optode. A flow cell can be mounted onto the sensing end of the optode for bringing samples, calibration or rinsing solutions to the substrate. In situ fluidic line is based on a peristaltic ten channels pump which is actuated by an oil pressure compensated electric motorization. One channel of this pump is connected on one side to the Raman probe and on the other side to a six-position switching valve purchased from Rheodyne. Three of the six valve inputs are connected to bags in which are stored pure water, ethanol and water solution of bi-pyridyl-ethylene (BPE). 


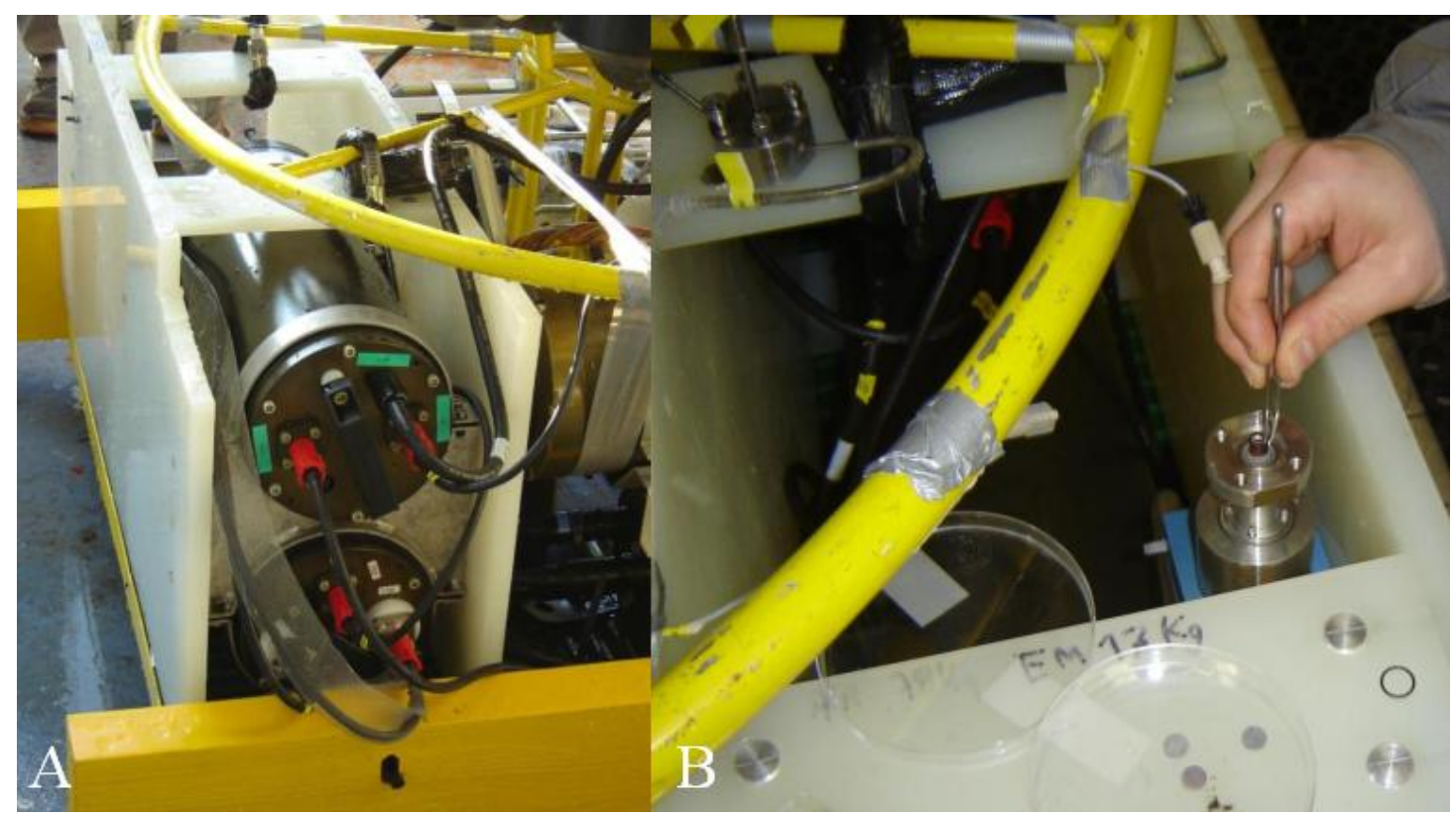

Figure 1: (A) In situ Raman spectrometer in operational configuration. (B) SERS substrate loading in the flow cell.

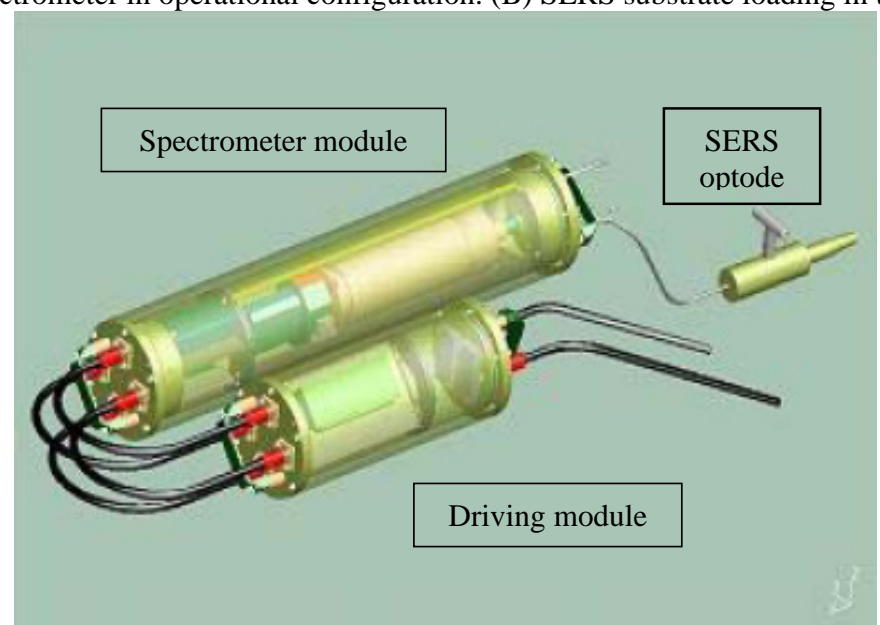

Figure 2: Raman spectrometer for in situ analysis with its three modules.

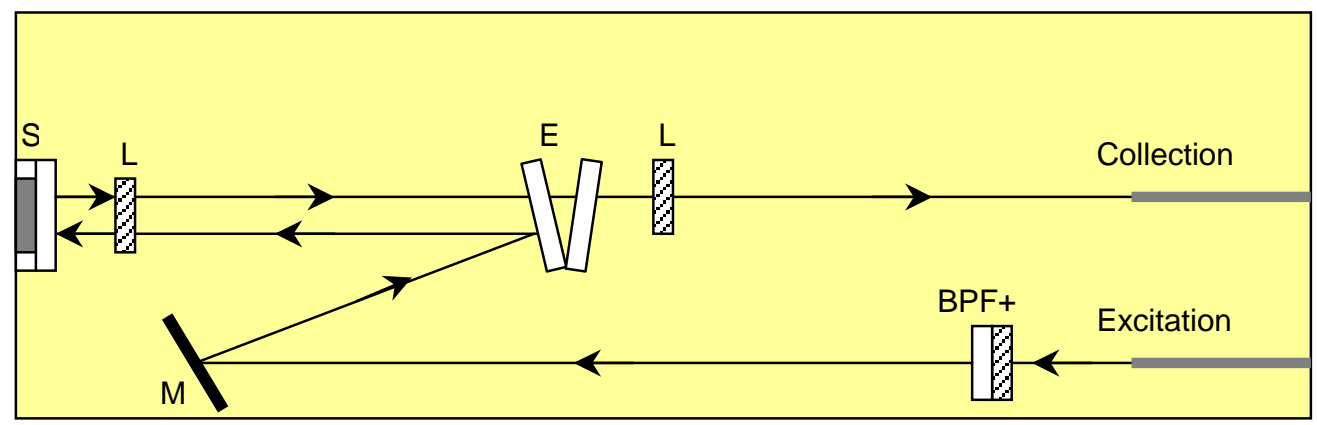

Figure 3 : Optical path within the optode. The light coming out of the excitation fiber is collimated by a lens (C) before going through a band-pass filter (BPF). Then, the laser beam is reflected first onto a dielectric miror (M) and secondly onto an edge filter (EF). It is then focused onto the SERS substrates (S) by the lens (L1). The back-scattered light is collected by the lens (L1) and goes through two edge filters (EF) that reflect the Rayleigh scattered light. A lens (L2) then launches the light into the collection fiber. 
Concerning colloidal film extinction spectra, a Raman spectrometer, without any edge filter, was used with a white exciting light through a lens $(\mathrm{x} 50$, NA $=0.75)$. In order to record the spectrum baseline, the bare substrate was illuminated.

Scanning electron microscopy (SEM) measurements were done with a field emission scanning electron microscope (FEIQuanta200). Before loading into the observation chamber, samples were coated with thin Au film ( 10-15 nm) by sputtering to avoid surfaces charging effect.

\subsection{SERS sensors}

Colloidal suspensions of gold nanoparticles, allowing SERS effect, were prepared according to the method described by Frens [18]. Such a metal preparation technique has been used in different studies [17,19-20] and consists in the reduction of $\mathrm{HAuCl}_{4}$ by sodium tricitrate. Two ways were considered to synthesize hydrophobic surfaces binding gold metal nanoparticles.

On the one hand, a silanization procedure was employed: clean disk quartz substrates were immersed in a $5 \%\left(\mathrm{v} \mathrm{v}^{-1}\right)$ methanol solution of MPMS or APTMS for four hours according to the results obtained by Seitz et al. [19], rinsed several times in methanol, sonicated in methanol for twenty minutes to remove the silane excess and dried for one hour at $100^{\circ} \mathrm{C}$ in an air oven.

On the other hand, a functionalization [21] procedure was used: disk quartz substrates coated by physical deposition with a thin film 1nm Cr / 50nm Au were immersed in a 5\% $\left(\mathrm{v} \mathrm{v}^{-1}\right)$ methanol solution of MPMS for three hours, rinsed in methanol, dipped two hours into a $0.01 \mathrm{M} \mathrm{NaOH}$ solution in order to interconnect the silanes between them and immersed again into the $5 \%\left(\mathrm{v} \mathrm{v}^{-1}\right)$ methanol solution of MPMS overnight.

In both cases, silanized and functionalized substrates were immersed into a colloidal suspension of gold nanoparticles for one hour and dried ten minutes at $100^{\circ} \mathrm{C}$. In order to reach the most favorable conception recipe, some substrates were kept two days in the air before immersion in the colloidal solution. Suitable sensors for adsorbed hydrophobic molecule species and SERS measurements were thus synthesized. Scheme 1 and scheme 2 present respectively silanization and functionalization representations of the SERS substrate fabrication procedures.

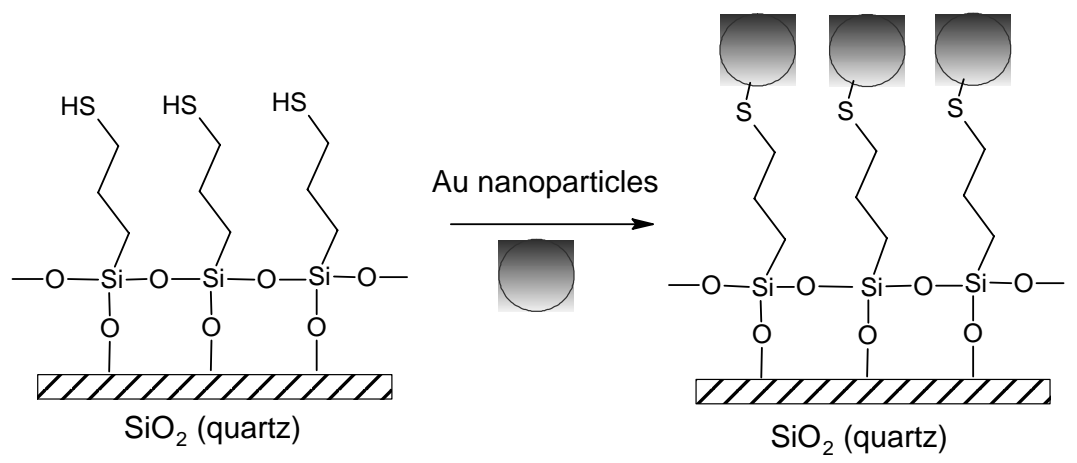

Scheme 1: Schematic representation of the SERS substrate silanization procedure (MPMS) and immersion in gold colloidal particles. 

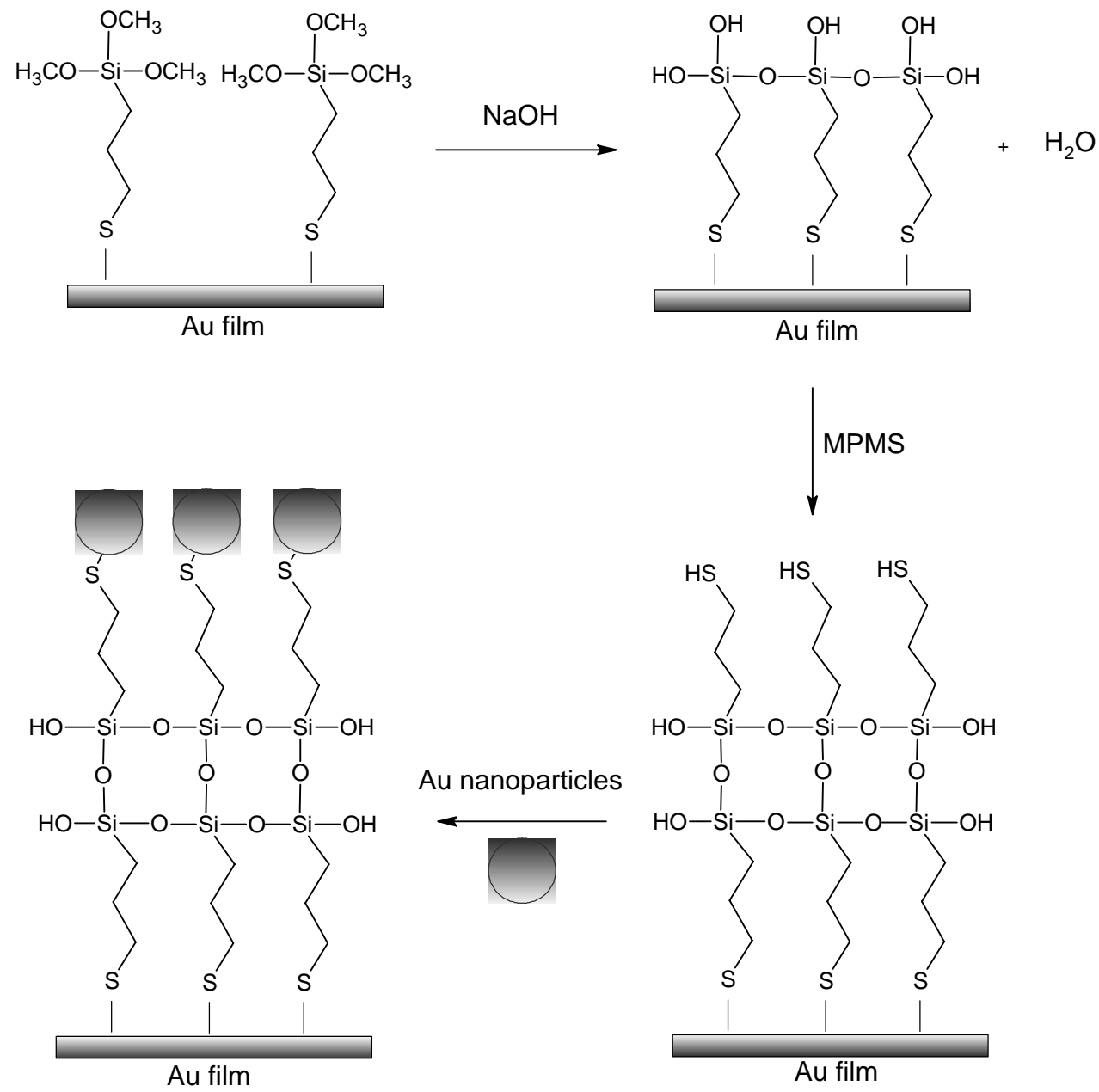

Scheme 2: Schematic representation of the SERS substrate functionalization procedure and immersion in gold colloidal particles.

\subsection{Targeted solutions}

Polycyclic aromatic hydrocarbon (PAH) solutions in artificial sea-water (ASW) were obtained. The naphthalene solution and the pyrene solution were prepared by dissolving the $\mathrm{PAH}$ in methanol wherein it is more soluble and diluted in ASW. ASW was prepared according to the ASTM D1141-90 norm [22]. Naphthalene and pyrene in pure water at $25^{\circ} \mathrm{C}$ are respectively soluble at $31.8 \mathrm{mg} . \mathrm{L}^{-1}$ and $0.13 \mathrm{mg} . \mathrm{L}^{-1}$. A $10 \mathrm{ppm}$ naphthalene solution and a 100ppb pyrene solution both diluted to 10ppb were achieved.

The 10ppm naphthalene solution was prepared from $78 \mu \mathrm{L}$ of $0.05 \mathrm{~mol}^{-1} \mathrm{~L}^{-1}$ naphthalene methanol solution and the corresponding blank composed by $78 \mu \mathrm{L}$ of methanol completed by $50 \mathrm{~mL}$ of ASW.

The 100ppb pyrene solution was prepared from $5 \mu \mathrm{L}$ of $0.005 \mathrm{~mol}^{-1} \mathrm{~L}^{-1}$ pyrene methanol solution and the corresponding blank composed by $5 \mu \mathrm{L}$ of methanol completed by $50 \mathrm{~mL}$ of ASW.

A trans-1,2-bis(4-pyridil)ethylene (BPE) aqueous solution with a concentration of $2.10^{-3} \mathrm{~mol} . \mathrm{L}^{-1}$ was also prepared. The BPE is currently used by the SERS community as molecule probe [23]. 


\section{RESULTS AND DISCUSSION}

Hydrophobic molecule pre-concentrations on nonpolar surfaces result in a physisorption process. The functional amine group with the free doublet of the APTMS confers a sizeable polarity to the substrate surface. Whereas the MPMS substrates may be more sensitive towards hydrophobic molecule pre-concentrations due to stronger hydrophobic interactions. In order to evaluate the detection reversibility of sensors and optimal organosilane silanization, tests were conducted on APTMS and MPMS SERS-active substrates. The high MPMS substrate predisposition in detecting and preconcentrating hydrophobic molecules was demonstrated [24].

The obtained sensors thanks to MPMS silanization procedure allowed a detection of PAH traces in artificial sea water (ASW). SERS substrate sensitivity was investigated by SERS measurements in ASW of naphthalene and pyrene solutions. The experiments were conducted on MPMS silanized substrates kept in the air for two days during their preparation before being immersed in colloidal suspension. These SERS-active substrates present extinction spectra with a broad plasmon band (600-700nm) (Figure 4 A) MPMS (b)) compare to those directly immersed into a colloidal suspension of gold nanoparticles (Figure 4 A) MPMS (a)). With a 632.8nm excitation line, such SERS substrates match the required conditions for detecting hydrophobic molecule traces until 10ppb [24]. Between each concentration test, the samples were rinsed with ethanol and blank measurements were achieved in order to confirm sensor reversibility. The SEM image of the sample shows a dense nanoparticle distribution with a couple of nanoparticles of large diameters. Size particles are in the range of 40-100nm. The results are presented Figure 4.

A)
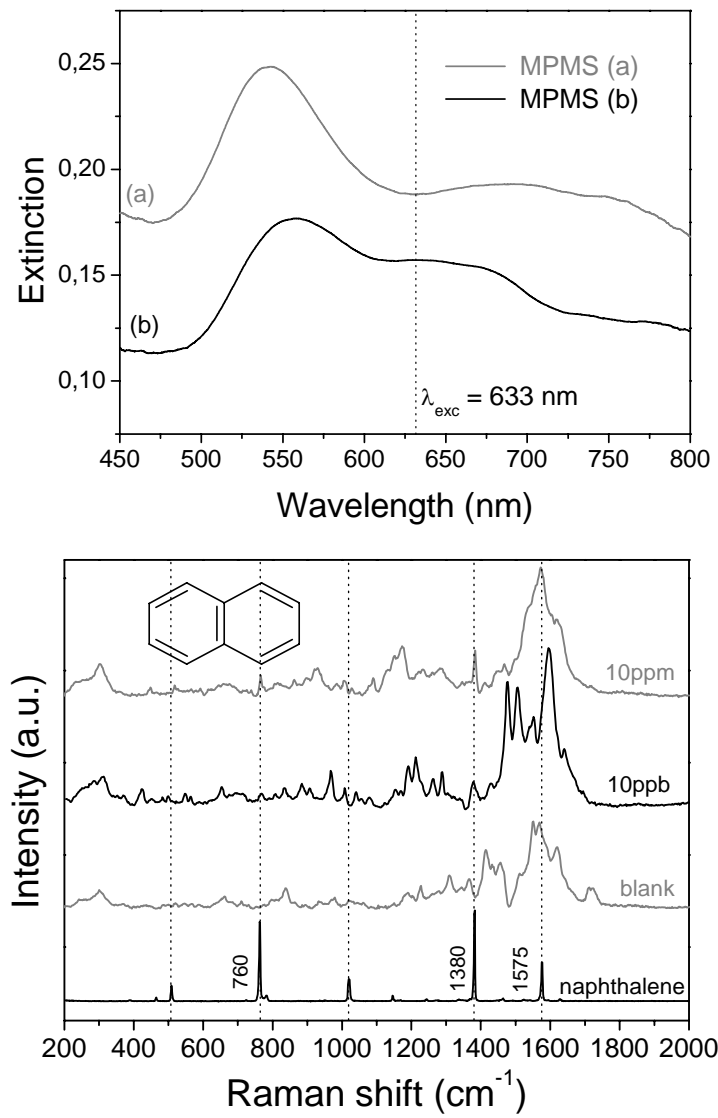

B)
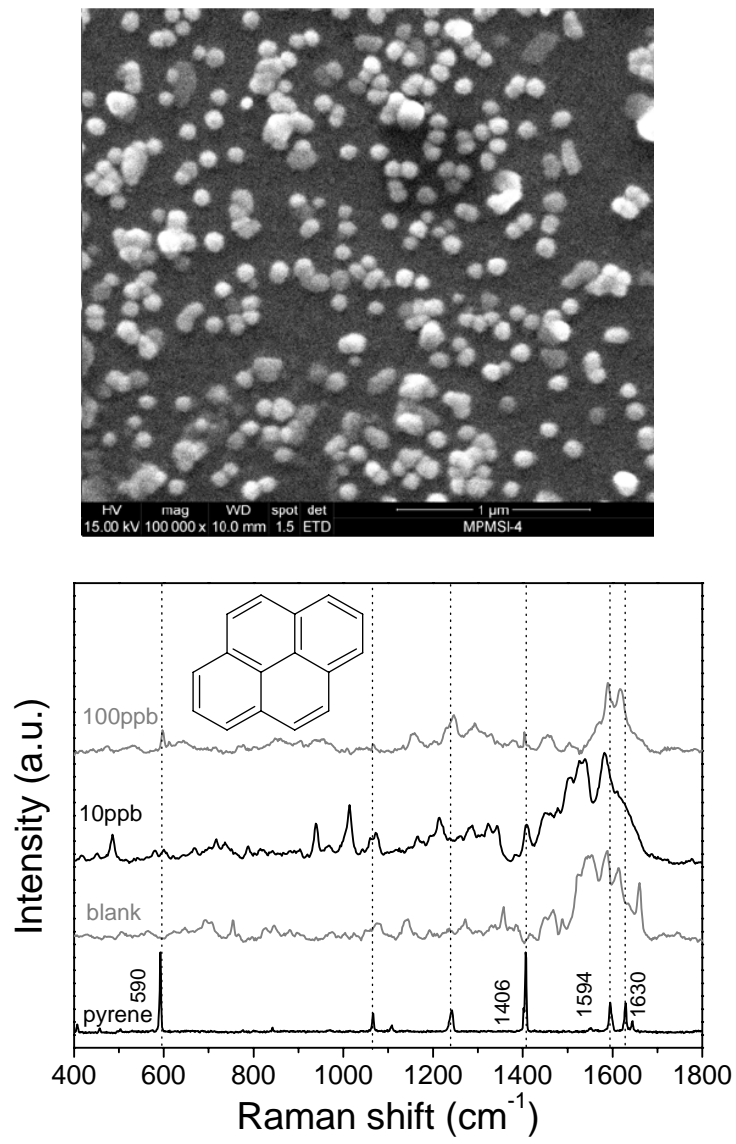

Figure 4: A) Extinction spectra of MPMS silanized substrates (a) directly immersed into a colloidal suspension of gold nanoparticles and (b) kept in the air for two days during their preparation before the immersion. All spectra were smoothed by adjacent averaging method (250 points). B) SEM image (x100 000) of SERS substrates (b).

C) SERS spectra of artificial sea-water naphthalene solutions at 10ppb and 10ppm, blank and for comparison Raman spectrum of solid-state naphthalene is also given. Integration time of 10s. D) SERS spectra of artificial sea-water pyrene solutions at 10ppb and $10 \mathrm{ppm}$, blank and for comparison Raman spectrum of solid-state pyrene is also given. Integration time of 10s. 
Nevertheless, such a silanization procedure presents one disadvantage. Even though the bare quartz cleaning is always the same and in consequence the density of hydroxyl group at the substrate surface is similar between each sample, the silanization reproducibility was not always obtained. Some bare substrates can not be silanized with success and the gold nanoparticles of the colloidal suspension can not be grafted. The alternative is the functionalization of quartz disk substrates coated by physical deposition with a thin film $1 \mathrm{~nm} \mathrm{Cr} / 50 \mathrm{~nm}$ Au. A thin film of chrome of $1 \mathrm{~nm}$ thickness is necessary in order to stick the gold metal onto the quartz substrate. By this way, the MPMS grafting is independent of the hydroxyl group density at the surface of the bare substrate. The affinity with gold metal of the - SH terminal group of the MPMS allows it chemical bond with quartz disk substrates coated by physical deposition.

SERS spectra of sensors obtained in the case of functionalization were recorded at different naphthalene concentrations in ASW. Between each concentration test, the samples were rinsed with ethanol and blank measurements were achieved in order to confirm sensor reversibility. The spectra were recorded with the $632.8 \mathrm{~nm}$ and $784.35 \mathrm{~nm}$ excitation lines from different spots on the substrate surface to promote SERS response homogeneity. A limit of detection (LOD) of 10ppb for naphthalene was determined (Figure 5).
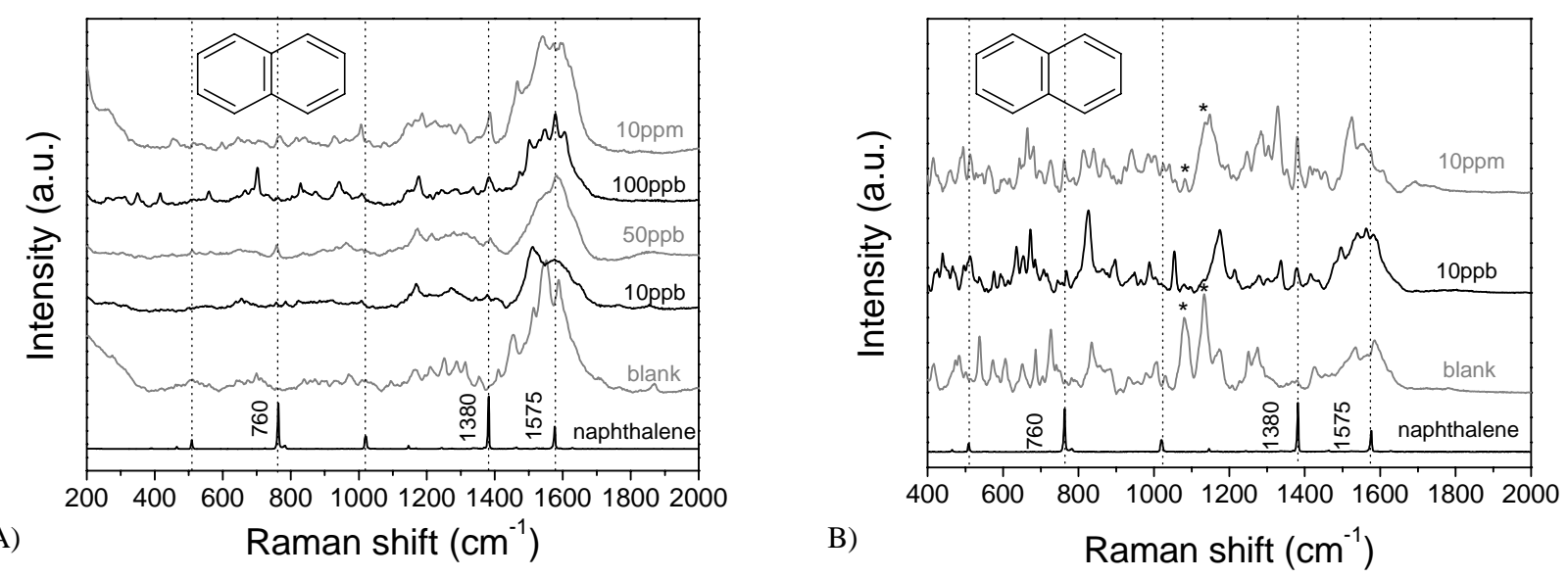

Figure 5: A) SERS spectra of artificial sea-water naphthalene solutions from 0 to 10ppm recorded with a 632.8nm excitation line and for comparison Raman spectrum of solid-state naphthalene is also given. Integration time of 5s. B) SERS spectra of artificial sea-water naphthalene solutions at 10ppb and 10ppm, blank recorded with a 784.38nm excitation line and for comparison Raman spectrum of solid-state naphthalene is also given. Integration time of 5s. The stars (*) indicate the sulfate ion vibrations.

Concerning the SERS results obtained in both cases, silanization (see Figure 4 C), D)) and functionalization (see Figure 5 A), B)), PAHs dissolved in ASW solution exhibit a random intensity variation of the main characteristic Raman peak. A well known theory [25], which stipulates that the vibrational parallel modes to the plane of the surface are the least enhanced modes, could explain the peak intensity variations. According to the surface selection rule and the adsorbate vibrational mode, different orientations and tilts of PAH molecules on substrate surface could occur [26]. In addition, the signature of the organic part evolves under laser excitation. The origin remains uncertain but during the reduction process of gold nanoparticles, sodium tricitrate excess and oxidation products still remain in the colloids. The displacement of these species arising from competitive adsorption on the metal surface could be responsible for changes in the spectra features [27]. Moreover, chemical oxidation phenomena of PAHs could occur in the case of PAH adsorption on gold island films [28]. Here, SERS substrate surfaces present a different catalytic activity as regards chemical species with free spaces on their surfaces. In our case, the intensity of the extinction spectra and of the laser power are lower. In the Figure $5 \mathrm{~B}$ ), the SERS spectra recorded under a 784.38nm excitation line seem to present an higher intensity of the Raman peaks for the blank spectra as well as for the naphthalene detection spectra. As we have first-mentioned in this paragraph, such peaks could correspond to the inorganic part or for example the Raman peaks indicated with a star $\left(^{*}\right)$ on the spectrum could be attributed to the sulphate ion vibrations which present a band at $1080-1130 \mathrm{~cm}^{-1}$ [29]. The sodium sulfate is one of the main molecular salt of the ASW.

In silanization and functionalization cases, the LODs of 10ppb for both PAHs, naphthalene and pyrene in ASW, were measured. Concerning naphthalene trace detection, the main peaks centered on $760 \mathrm{~cm}^{-1}$ and $1380 \mathrm{~cm}^{-1}$ are preferentially 
considered. In the pyrene case, peaks centered on $590 \mathrm{~cm}^{-1}$ and $1406 \mathrm{~cm}^{-1}$ with a higher intensity were preferentially studied. The peaks at $1575 \mathrm{~cm}^{-1}$ for naphthalene, $1594 \mathrm{~cm}^{-1}$ and $1630 \mathrm{~cm}^{-1}$ for pyrene can not be easily analyzed since the organic part, i.e. MPMS and sodium tricitrate excess, presents Raman signals in this spectral region. Such results confirm SERS MPMS substrate performance in relation to hydrophobic sensitivity and sensors. We can not draw any conclusions on the evolution of the spectral intensity of the Raman peaks versus PAH concentration in so far as isolated sites on the surface, called "hot spots", may give rise to intense local plasmon fields. Moreover, 10ppb LODs in ASW for both PAHs with an integration time of 10 s could lead to a more sensitive detection by increasing the integration time of the experiment. In this way a sub-ppb detection sensor may be obtained with a reasonable resolution of signal/noise (> 10) on the main characteristic Raman peaks.

In parallel to these laboratory studies, shipboard experiments were conducted. The major goal was to study the feasibility of SERS measurement in a flow cell in seawater. APTMS SERS substrates were used. Bi-pyridyl-ethylene (BPE) solution at $10^{-3} \mathrm{M}$ has been selected as a molecular probe to reveal SERS activity of sensors. During in situ experiments, pure water was firstly introduced in the flow cell on the substrate, and afterwards BPE. Ethanol was used in order to remove adsorbed BPE. Figure 6 shows therefore a Raman spectrum of both BPE and ethanol. The bands located at 810, 895 (strong), and $1460 \mathrm{~cm}^{-1}$ marked with diamonds on Figure 6 are assigned to ethanol; this signal is probably not exalted and corresponds only to Raman scattering through liquid ethanol in the cell. However, three doublets and single band marked with stars and located at 1615-1640 / 1350-1390/1210-1255 cm and $1030 \mathrm{~cm}^{-1}$ are assigned to BPE. The other bands are assigned to the organic matter from APTMS silanization.

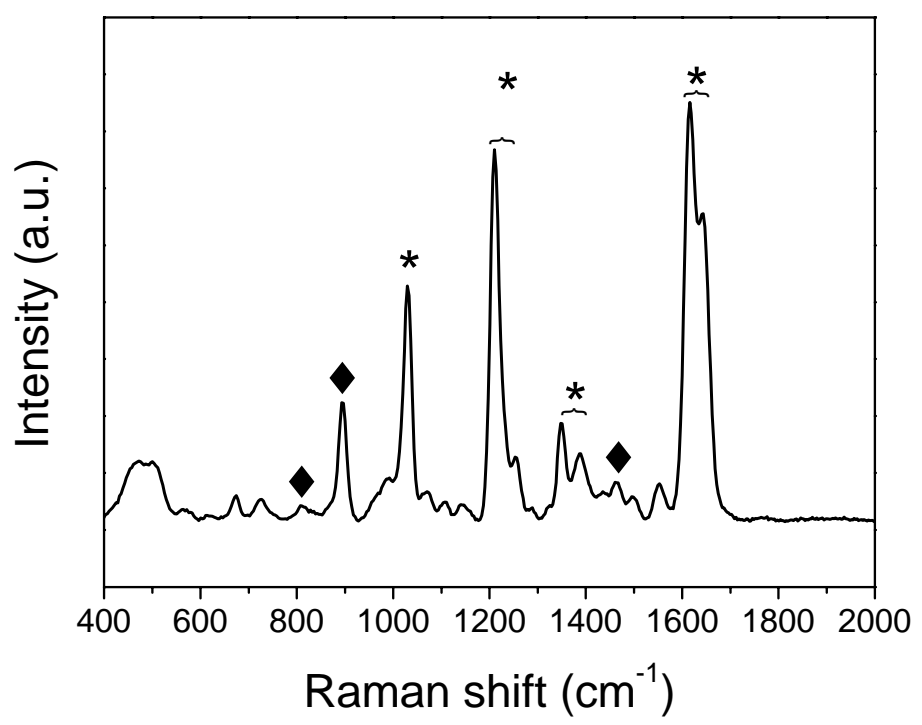

Figure 6: Raman spectrum obtained during shipboard experiments. Diamonds correspond to ethanol bands and stars to BPE.

\section{CONCLUSION}

The development of a SERS sensor for analyses in sea-water has been described in terms of chemical synthesis and optical development. Based on gold nanostructures, different substrates were synthesized to modify some parameters such as gold shape and distribution and such as chemical functionalization. Low concentration from ppb to ppm of pyrene and naphthalene were detected in ASW with a short integration time of 10s and under a low incident laser power of approximately $0.1 \mathrm{~mW}$. The home-made in situ Raman spectrometer connected to a micro-fluidic system has permitted original SERS measurements of BPE with APTMS substrates. This original configuration of in situ Raman spectroscopy is now ready to confirm PAHs detection at ppb levels during future offshore experiments thanks to new MPMS SERS 
sensors. In order to well control the SERS response of the sensors, works are in progress concerning the fabrication of nanostructures of a desired size, shape and arrangement on quartz disk substrates through electron beam lithography (EBL) using the lift-off technique. Such a technique allows a reproducibility of SERS substrates. By this way the SERS response of the sensors evolves in accordance with the excitation line and the nanoparticle diameter can be adapted to the laser excitation.

\section{AKNOWLEGDEMENT}

This project was supported by ANR financing (P2IC: Discomar) and carried out in conjunction with the Laboratoire de Nanotechnologie et d'Instrumentation Optique (LNIO) - Université de Technologie de Troyes (UTT).

The authors acknowledge Philippe Crassous, IFREMER - Laboratoire Environnement Profond at Plouzané France, for the SEM images.

\section{REFERENCES}

[1] Moskovits, M., “Surface-enhanced spectroscopy”, Rev. Mod. Phys. 57, 783-826 (1985).

[2] Otto, A., "Surface-enhanced Raman of adsorbate”, J. Raman Spectrosc. 22, 743-752 (1991).

[3] Otto, A., Mrozek, I., Grabhorn, H. and Akemann W., "Surface-enhanced Raman-scattering”, J. Phys. Condens. Matter. 4, 11431212 (1992).

[4] Fleischmann, M., Hendra, P.J., McQuillan, A.J., Paul, R.L. and Reide, E.S., "Raman spectroscopy at electrode-electrolyte interfaces”, J. Raman Spectrosc. 4, 269-274 (1976).

[5] Jeanmaire, D.J. and Van Duyne, R.P., "Surface Raman spectroelectrochemistry Part I. Heterocyclic, aromatic, and aliphatic amines adsorbed on the anodized silver electrode”, J. Electronanal. Chem. 84, 1-20 (1977).

[6] Albrecht, M.G. and Creighton, J.A., “Anomalously intense Raman spectra of pyridine at a silver electrode”, J. Am. Chem. Soc. 99, 5215-5217 (1977).

[7] Nie, S. and Emory, S.R., "Probing single molecules and single nanoparticles by surface-enhanced Raman scattering”, Science 275, 1102-1106 (1997).

[8] Kneipp, K., Wang, Y., Kneipp, H., Perelman, L.T., Itzkan, I., Dasari, R.R. and Feld, M.S., "Single molecule detection using surface-enhanced Raman scattering”, Phys. Rev. Lett. 78, 1667-1670 (1997).

[9] Kneipp, K., Kneipp, H., Deinum, G., Itzkan, I., Dasari, R.R. and Feld, M.S., "Single molecule detection of a cyanine dye in silver colloidal solution using near infrared surface-enhanced Raman scattering”, Appl. Spectrosc. 52, 175-178 (1998).

[10] Kneipp, K., Kneipp, H., Itzkan, I., Dasari, R.R. and Feld, M.S., "Surface-enhanced non-linear Raman scattering at the single molecule level”, Chem. Phys. 247, 155-162 (1999).

[11] Murphy, T., Schmidt, H. and Kronfeldt, H.D., "Detection of chemicals in sea-water using surface enhanced Raman scattering (SERS)", SPIE 3107, 281-287 (1997).

[12] Murphy, T., Schmidt, H. and Kronfeldt, H.D., "Use of sol-gel techniques in the development of surface-enhanced Raman scattering (SERS) substrates suitable for in situ detection of chemicals in sea water”, Appl. Phys. B 69, 147-150 (1999).

[13] Lucht, S., Murphy, T., Schmidt, H. and Kronfeldt, H.D., “Optimized recipe for sol-gel-based SERS substrates”, J. Raman Spectrosc. 31, 1017-1022 (2000).

[14] Schmidt, H., Bich Ha, N., Pfannkuche, J., Amann, H., Kronfeldt, H.D. and Kowalewska, G., "Detection of PAHs in seawater using surface-enhanced Raman scattering (SERS)”, Mar. Poll. Bull. 49, 229-234 (2004).

[15] Nuzzo, R.G., Zegarski, B.R. and Dubois, L.H., "Fundamental studies of the chemisorption of organosulfur compounds on Au (111). Implications for molecular self-assembly on gold surfaces”, J. Am. Chem. Soc. 109, 733-740 (1987).

[16] Grabar, K.C., Allison, K.J., Baker, B.E., Bright, R.M., Brown, K.R., Freeman, R.G., Fox, A.P., Keating, C.D., Musick, M.D. and Natan, M.J., "Two-dimensional arrays of colloidal gold particles: a flexible approach to macroscopic metal surfaces”, Langmuir 12, 2353-2361 (1996).

[17] Keating, C.D., Musick, M.D., Keefe, M.H. and Natan, M.J., "Kinetics an thermodynamics of au colloid monolayer selfassembly”, J. Chem. Educ. 76, 949-955 (1999).

[18] Frens, G., "Preparation of gold dispersions of varying particle size: controlled nucleation for the regulation of the particle size in monodisperse gold suspensions”, Nature, Phys. Sci. 241, 20-22 (1973).

[19] Seitz, O., Chehimi, M.M., Cabet-Deliry, E., Truong, S., Felidj, N., Perruchot, C., Greaves, S.J. and Watts, J.F, "Preparation and characterization of gold nanoparticles assemblies on silanised glass plate”, Colloids Surf. A: Physicochem. and Eng. Aspects 218, 225-239 (2003).

[20] Lee, P.C. and Meisel, D., “Adsorption and surface-enhanced Raman of dyes on silver and gold sols”, J. Phys. Chem. 86, 33913395 (1982).

[21] Fu, Y., Yuan, R., Xu, L., Chai, Y., Zhong, X. and Tang, D., "Indicator free DNA hybridization detection via EIS based on selfassembled gold nanoparticles and bilayer two-dimensional 3-mercaptopropyltrimethoxysilane onto a gold substrate”, Biochem. Eng. J. 23, 37-44 (2005). 
[22]American Society for Testing Materials, "Designation D 1141-90 (reapproved 1992)", ASTM,(1992).

[23] Yang, W., Hulten, J., Schatz, G.C. and Van Duyne, R.P., "A surface-enhanced hyper Raman and surface-enhanced Raman scattering study of trans-1,2bis(4-pyridyl)ethylene adsorbed onto silver film over nanosphere electrodes. Vibrational assignments: experiment and theory”, J. chem. Phys. 104, 4313-4323 (1996).

[24] Péron, O., Rinnert, E., Lehaitre, M., Crassous, P. and Compère, C., "Detection of polycyclic aromatic hydrocarbon (PAH) compounds in artificial sea-water using Surface-Enhanced Raman Scattering (SERS)", accepted in Talanta, (2009).

[25] Creighton, J.A., "Surface Raman electromagnetic enhancement factors for molecules at the surface of small isolated metal spheres: the determination of adsorbate orientation from SERS relative intensities”, Surf. Sci. 124, 209-219 (1983).

[26] Lee, T.G., Kim, K. and Kim, M.S., “Adsorption of xylene- $\alpha$, $\alpha$ '-dithiol on silver investigated by Raman spectroscopy”, J. Phys. Chem. 95, 9950-9955 (1991).

[27] Sanchez-Cortés, S.and Garcia-Ramos, J.V., “Anomalous Raman bands appearing in surface-enhanced Raman spectra”, J. Raman Spectrosc. 29, 365-371 (1998).

[28] Costa, J.C.S., Sant’Ana, A.C., Corio, P. and Temperini, M.L.A., “Chemical analysis of polycyclic aromatic hydrocarbons by surface-enhanced Raman spectroscopy”, Talanta 70, 1011-1016 (2006).

[29] Socrates, G., "Infrared and Raman characteristic group frequencies”, John Wiley \& Sons Ltd, 277-285 (2001). 